DOI: $10.15593 / 2224-9982 / 2019.57 .07$

УДК $62-135.3$

\author{
В.И. Милешин, А.М. Петровичев, С.И. Баева, В.В. Жданов \\ Центральный институт авиационного моторостроения им. П.И. Баранова (ЦИАМ), \\ Москва, Россия \\ РАСЧЕТНО-ЭКСПЕРИМЕНТАЛЬНОЕ ИССЛЕДОВАНИЕ \\ ВЛИЯНИЯ НАДРОТОРНЫХ УСТРОЙСТВ ЛАБИРИНТНОГО ТИПА \\ НА ХАРАКТЕРИСТИКИ ЗАМЫКАЮЩЕЙ СТУПЕНИ КОМПРЕССОРА
}

\begin{abstract}
Высокие степени повышения давления в современных осевых компрессорах приводят к уменьшению высоты проточной части и, следовательно, к уменьшению высоты лопаток в последних ступенях компрессора. Зазор на периферии лопатки не может быть снижен по отношению к высоте или длине хорды лопатки на такую же относительную величину, что и в первых ступенях. Более того, работа компрессора на переходных режимах может привести к увеличению зазоров на периферии, так что в последних ступенях значения зазоров будут заметно выше обычных. Это может повлиять на диапазон рабочих режимов и характеристики всего компрессора. Надроторное устройство лабиринтного типа может быть одним из средств парирования негативных последствий, связанных с увеличением зазора на перифрерии ротора.

Объектом исследования данной работы является ступень Д-77М - крупногабаритная (1 м) модель последней ступени КВД, предназначенная для исследования особенностей течения в осевых ступенях с большим относительным диаметром втулки $\bar{d}=0,925$. Она состоит из трехлопаточных венцов: входной направляющий аппарат с числом лопаток $Z_{\mathrm{BHA}}=90$, который создает закрутку, подобно закрутке в реальном компрессоре, рабочее колесо с числом лопаток $Z_{\mathrm{PK}}=82$ и двухрядный направляющий аппарат с числом лопаток $Z_{\mathrm{HA}}=134+134$. Ступень Д-77М имеет следующие проектные параметры: $G_{\text {пр }}=15,4 \mathrm{kr} / \mathrm{c} ; u_{\text {пр }}=264 \mathrm{~m} / \mathrm{c} ; \pi^{*}=1,24$.

Целью проектирования бороздок НРУ лабиринтного типа было восстановить запасы газодинамической устойчивости, величину степени повышения полного давления и максимальный уровень КПД, которые уменьшились из-за увеличения радиального зазора. Были рассмотрены два значения радиального зазора: a) $d_{p}=0,4$ мм - проектная высота радиального зазора и б) $d_{p}=0,8$ мм - увеличенная величина радиального зазора.

Ключевые слова: замыкающая ступень компрессора, надроторное устройство лабиринтного типа, радиальный зазор, КПД, газодинамическая устойчивость.
\end{abstract}

\author{
V.I. Mileshin, A.M. Petrovitchev, S.I. Baeva, V.V. Zhdanov
}

Central Institute of Aviation Motors named after P.I. Baranov (CIAM), Moscow, Russian Federation

\title{
NUMERICAL AND EXPERIMENTAL STUDY OF THE EFFECT OF CIRCUMFERENTIAL GROOVES ON HPC LAST STAGE PERFORMANCE
}

High pressure ratios in advanced axial compressors lead to a decrease in height of the flow path and, consequently, a decreased blade height in HPC last stages. The blade tip clearance can't be reduced relatively to the blade chord length or height by the same relative value as in first stages. Moreover, HPC operation in transient conditions can cause an increase in tip clearances, so that clearances in last stages can exceed normal values. This may affect the operating range and characteristics of the compressor as a whole. Circumferential groove casing treatments can be used to compensate for negative effects associated with an increase in tip clearances.

The test unit used in this work is a D-77M stage - the large-scale $(1 \mathrm{~m})$ model of the HPC last stage designed for studies of flow specifics in axial stages with a big hub-to-tip ratio equal to 0.925 . It consists of 3 blade rows: inlet guide vanes (IGV) with 90 vanes (ZIGV), which provide the same flow swirling as in an actual compressor, a rotor with 82 blades (ZR) and a tandem stator with $134+134$ vanes (ZSII). The D-77M stage has the following design parameters: mass air flow $15.4 \mathrm{~kg} / \mathrm{s}$; tip speed $264 \mathrm{~m} / \mathrm{s}$; total pressure ratio 1.24 .

The purpose of the circumferential grooves is recovery the stall margin, pressure ratio and maximum level of efficiency, which have decreased due to an increase in the tip clearance. Two values of the tip clearance are studied: a) $0.4 \mathrm{~mm}-$ design value of the tip clearance, and b) $0.8 \mathrm{~mm}$ - increased value of the tip clearance.

Keywords: compressor last stage, circumferential grooves casing treatment, radial clearance, adiabatic efficiency, stall margin. 


\section{Условные обозначения}

Термодинамические и газодинамические параметры:

$P \quad$ - давление, Па;

$T$ - абсолютная температура, К;

$G \quad$ - массовый расход воздуха, кг/с;

$\pi^{*} \quad$ - степень повышения полного давления;

$\eta_{\text {ад }}^{*} \quad$ - адиабатический коэффициент полезного действия, $\eta_{\text {ад }}^{*}=H_{\text {ад }} / H_{Z}$.

Геометрические параметры:

$D, R$ - диаметр, радиус, м;

$\bar{d}$ - относительный диаметр втулки;

$d_{\mathrm{p}} \quad$ - радиальный зазор, мм;

$z \quad$ - количество бороздок в НРУ;

Z - количество лопаток.

Кинематические параметры:

n - частота вращения ротора, об/мин;

$u$ - окружная скорость ротора, м/с;

$C$ - абсолютная скорость потока (в неподвижной системе координат), м/с;

$W$ - относительная скорость потока.

Индексы и сокращения:

ВНА - входной направляющий аппарат;

КПД - коэффициент полезного действия;

РК - рабочее колесо (ротор);

$\mathrm{CA}_{\text {II }}$ - двухрядный выходной спрямляющий аппарат (статор);

НРУ - надроторное устройство;

СК - система координат;

п - периферийный;

к - компрессор;

вт - втулка;

пр - приведенный.

\section{Введение}

В связи с разработкой нового семейства высоконапорных КВД с ультравысокой степенью повышения полного давления на $\pi_{\mathrm{k}}^{*}=23 . .27$ при числе ступеней $z=10 \ldots 1$ [1-3] особое значение приобретает влияние радиального зазора в группе последних ступеней КВД [1, 4, 5]. Увеличение радиального зазора относительно проектной величины приводит к значительному ухудшению характеристик КВД. Для парирования негативного влияния радиального зазора на внутреннюю поверхность корпуса КВД наносится легко срабатываемое покрытие. Однако в процессе эксплуатации КВД из-за наличия переходных нестационарных режимов, овализации корпусов, входной неравномерности потока происходит выработка покрытия корпусов, которая приводит к увеличению величины радиального зазора и к дальнейшему ухудшению характеристик КВД [6-8].

В работе [9] исследовалось влияние НРУ лабиринтного типа на характеристики типовой средней ступени КВД при увеличенном радиальном зазоре. Показано, что из-за увеличения зазора над рабочим колесом падают запасы ГДУ, и применение НРУ лабиринтного типа позволяет устранить этот негативный эффект. Бороздки уменьшают поток массы через радиальный зазор и располагают траекторию вихря перетекания глубже в межлопаточном канале, что положительно влияет на запасы ГДУ.

Надроторные устройства лабиринтного типа могут оказывать существенное влияние на характеристики компрессора, при этом они достаточно просты в изготовлении. В последнее 
время было проведено большое количество работ, направленных на изучение механизма влияния таких НРУ. Хорошо известно, что бороздки позволяют воздействовать на течение перетекания через радиальный зазор, ослабляя его свертывание [10]. Полезность применения НРУ лабиринтного типа для увеличения запасов ГдУ была подтверждена в численном исследовании [11]. В работах [12-14] подчеркивается, что физический эффект от бороздок состоит в значительном уменьшении загромождения на периферии и ослаблении свертывания вихря перетекания через радиальный зазор.

В работе [15] исследовано влияние формы и положения одной бороздки на КПД и запасы ГДУ. Выделено два типа бороздок: бороздка треугольного сечения с углом скоса против потока, располагающаяся в области передней кромки лопатки рабочего колеса, которая положительно влияет на запасы при незначительном уменьшении КПД, и бороздка трапециевидного сечения, располагающаяся в области задней кромки, которая увеличивает КПД, но при этом негативно влияет на запасы устойчивости. В то же время показано, что эффекты от каждой из бороздок независимы и использование комбинации этих бороздок позволяет компенсировать потери от использования каждой из них по отдельности.

В работе [16] исследование проводилось при увеличенном зазоре над рабочим колесом. Было показано, что из-за изменения поля течения при увеличении зазора не сохраняется положительный эффект НРУ, который был получен при номинальном зазоре. Также отмечается, что важным параметром является осевое расположение бороздки.

В работе [17] исследовалось влияние положения НРУ лабиринтного типа, состоящего из шести прямоугольных бороздок. Рассматривалось три положения НРУ: в первом случае все бороздки находились над рабочим колесом, во втором и третьем вся комбинация бороздок была сдвинута вниз и вверх по потоку соответственно. Наибольший прирост запасов ГДУ был получен, когда все бороздки находились в области над рабочим колесом $(10,3 \%)$.

В работе [18] исследовалось влияние НРУ лабиринтного типа и изгиба периферии лопатки рабочего колеса на запасы ГДУ. Показано, что с помощью изгиба лопатки можно дополнительно увеличить запасы.

В работе [19] исследовалась комбинация НРУ щелевого и лабиринтного типов. В области передней кромки располагалось щелевое НРУ, а в области задней кромки - лабиринтное. В результате расчетов и экспериментов получилось, что проявляются эффекты обоих НРУ. Показано, что изменение запасов устойчивости связано с влиянием НРУ на траекторию вихря перетекания. Чем мощнее вихрь, тем сильнее его траектория стремится к стороне разряжения лопатки. Потери КПД связаны как с увеличением производства энтропии в щелях и бороздках НРУ, так и с потерями, вызванными течением, индуцированным щелями НРУ.

В настоящей работе все расчетные исследования были проведены на основе программного комплекса NUMECA Fine Turbo версия 10.1 [20, 21]. Для верификации математической модели и численного метода NUMECA Fine Turbo было проведено сопоставление расчетных и экспериментальных характеристик ступени Д-77М с гладкой проточной частью. Для построения сетки использовался сеточный генератор NUMECA Autogrid 5.

\section{Объект исследования}

Ступень Д-77М (рис. 1) является крупногабаритной моделью последней ступени КВД, предназначенной для исследования особенностей течения в осевых ступенях с большим относительным диаметром втулки $\bar{d}=0,925$, и состоит из трех лопаточных венцов: ВНА с числом лопаток $Z_{\mathrm{BHA}}=90$, РК с числом лопаток $Z_{\mathrm{PK}}=82$ и двухрядный $\mathrm{CA}$ с числом лопаток $Z_{\text {СAII }}=134+134$. Ступень имеет следующие расчетные параметры: $G_{\text {пр }}=15,4$ кг $/ \mathrm{c} ; u_{\text {пр }}=264 \mathrm{~m} / \mathrm{c}$; $\pi^{*}=1,24$. 


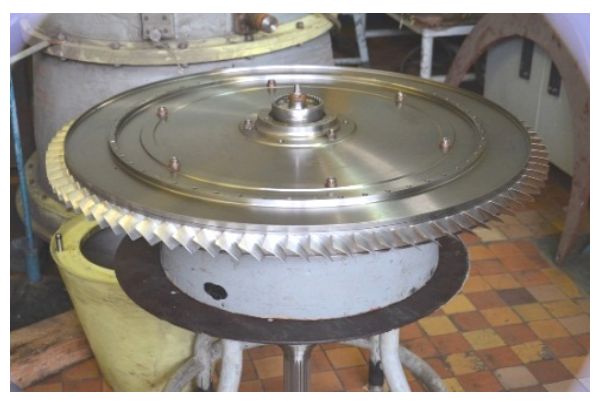

$a$

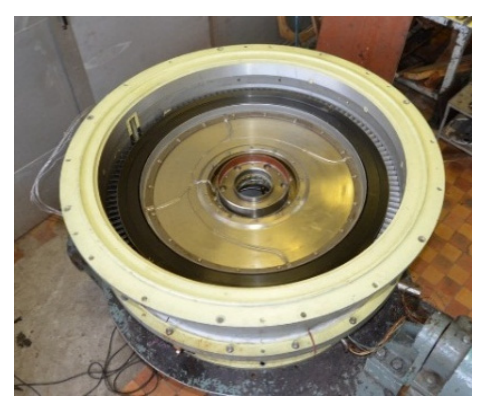

$\sigma$

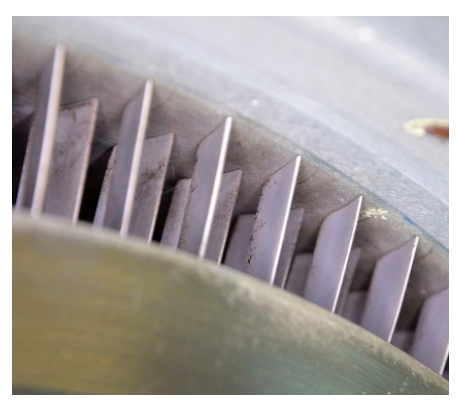

B

Рис. 1. Ступень D-77M: $a$ - рабочее колесо ступени Д-77М; $\sigma$ - выходной корпус; 6 - двухрядный CA

Конструкция ступени Д-77М разработана исходя из того, что она в основном предназначена для исследования влияния НРУ различного типа на характеристики типовой замыкающей ступени КВД с возможностью изменения радиального зазора над рабочим колесом.

В настоящей работе было исследовано два значения радиального зазора над рабочим колесом: рабочий зазор $d_{\mathrm{p}}=0,4$ мм и увеличенный зазор $d_{\mathrm{p}}=0,8$ мм. Изменение радиального зазора на ступени Д-77М производилось с помощью изменения диаметра наружного корпуса над рабочим колесом.

Для изменения радиального зазора и числа бороздок НРУ было изготовлено несколько съемных корпусов над РК, включая корпусы с гладкой проточной частью и с НРУ лабиринтного типа с числом бороздок $z=4$ и $z=6$.

Из-за технологических ограничений испытания были проведены только для НРУ с четырьмя бороздками без выходного двухрядного статора ступени Д-77М. С выходным статором испытания будут проведены на следующих этапах работы.

\section{Численная модель}

Численное моделирование течения выполнено с помощью программного комплекса NUMECA FINE TURBO 10.1 для решения осредненных по Рейнольдсу стационарных и нестационарных 3D-уравнений Навье - Стокса (RANS, URANS) [20, 21].

Работа была проведена в два этапа. Сначала для более детального исследования механизма влияния НРУ лабиринтного типа над РК использовались два венца: ВНА и РК. После этого был добавлен в расчет двухрядный выходной статор и проведены аналогичные исследования для полной ступени Д-77М.

На первом этапе сквозные расчеты вязких трехмерных течений в венцах выполнены в приближении mixing plane, для которого течения стационарны во вращающейся с каждым лопаточным венцом системе координат. Также для неполной ступени были получены нестационарные поля течения с использованием нелинейного гармонического метода (NLH). Основная идея этого метода заключается в том, что возмущения, делающие течение нестационарным, записываются осредненными по времени величинами и раскладываются в ряды Фурье. Представляя нестационарную систему уравнений Навье - Стокса в частотном виде, получаем уравнения переноса для каждой частоты. На интерфейсе между статором и ротором необходимо удовлетворить условию равенства решений с обеих сторон. Основная цель - получить непрерывное нестационарное течение через интерфейс. Поскольку число гармоник ограничено, непрерывность не может быть строго воспроизведена, но различия в численных результатах падают с ростом числа гармоник. В данном исследовании число гармоник принималось равным трем.

В качестве модели турбулентности для исследования влияния сетки и надроторных устройств используется двухпараметрическая модель турбулентной вязкости SST с использовани- 
ем пристеночных функций. Решения получены на основе конечно-разностной схемы Джеймсона второго порядка аппроксимации по пространству и времени.

На втором этапе из-за особенностей обтекания статора в качестве модели турбулентности используется двухпараметрическая модель турбулентной вязкости $k-\varepsilon$ с использованием пристеночных функций. Для полной ступени исследование ограничивается только подходом mixing plane.

При проведении расчетов использована блочно-структурированная сетка, построенная с помощью сеточного генератора Numeca Autogrid 5 (рис. 2-4). На первом этапе расчетная область состояла из 16 сеточных блоков в межлопаточных каналах (1627508 ячеек) и 4 или 6 блоков в НРУ (121 841 ячейка на одну бороздку четырехугольного сечения, 104673 ячейки на одну бороздку треугольного сечения). На втором этапе вся расчетная область разделена между 28 сеточными блоками (4959 728 ячеек) в межлопаточных каналах и 4 или 6 блоками в НРУ (121 841 ячейка на одну бороздку четырехугольного сечения, 104673 ячейки на одну бороздку треугольного сечения). Величина $y^{+} \sim 1$. Общий вид расчетной области изображен на рис. $2-4$.

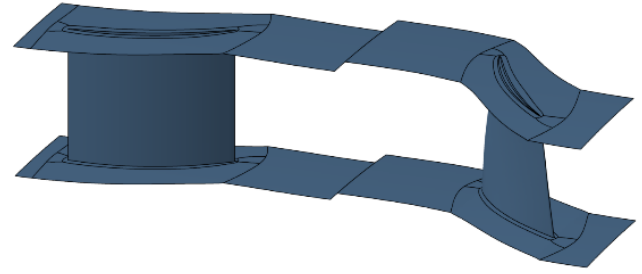

Рис. 2. Расчетная область ВНА + РК

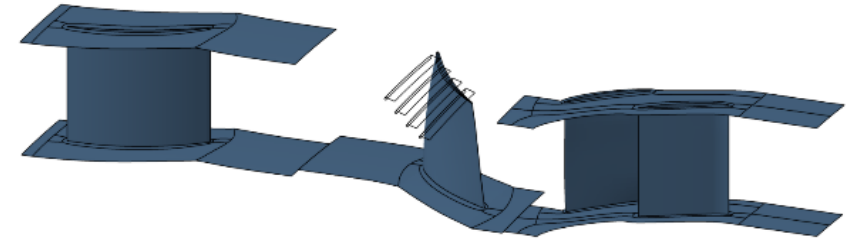

Рис. 3. Расчетная область ВНА + $\mathrm{PK}+\mathrm{CA}_{\mathrm{II}}$

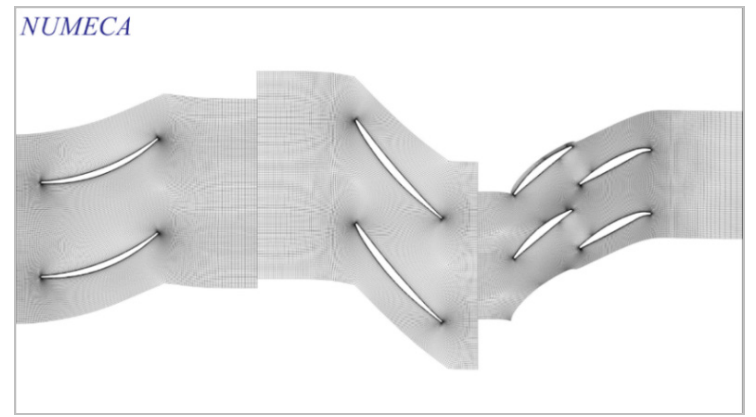

Рис. 4. Конечно-разностная сетка в сечении межлопаточного канала ступени Д-77М

Расчеты вязкого течения выполнены по приведенным параметрам: на входе $T=288,15 \mathrm{~K}$, $P=101325$ Па при оборотах $n=100 \%$, что составляет 5042 об/мин соответственно. В сечении выхода использовано условие радиального равновесия течения, фиксируется статическое давление на периферии. На твердых поверхностях заданы условия прилипания и отсутствия теплопередачи. Было рассмотрено два значения радиальных зазора на периферии рабочего колеса: $d_{\mathrm{p}}=0,4$ мм - проектная высота радиального зазора и $d_{\mathrm{p}}=0,8$ мм - увеличенная величина радиального зазора [22].

На основе результатов изученных работ была выбрана треугольная форма сечения бороздки, которые в количестве 6 штук были расположены над всей хордой лопатки. Ширина бороздки треугольного сечения - 3 мм, высота -2 мм, расстояние между бороздками - 1 мм. В дальнейшем, исходя из технологических возможностей реализации такого НРУ, было спроектировано урезанное сечение бороздки и уменьшено количество бороздок в НРУ (рис. 5). Ширина бороздки четырехугольного сечения - 3 мм, высота - 1 мм, расстояние между бороздками - 4 мм. Сеточные блоки, соответствующие окружным проточкам, имеют периодичность, равную периодичности лопаточных каналов рабочего колеса $(Z=82$ лопатки). 

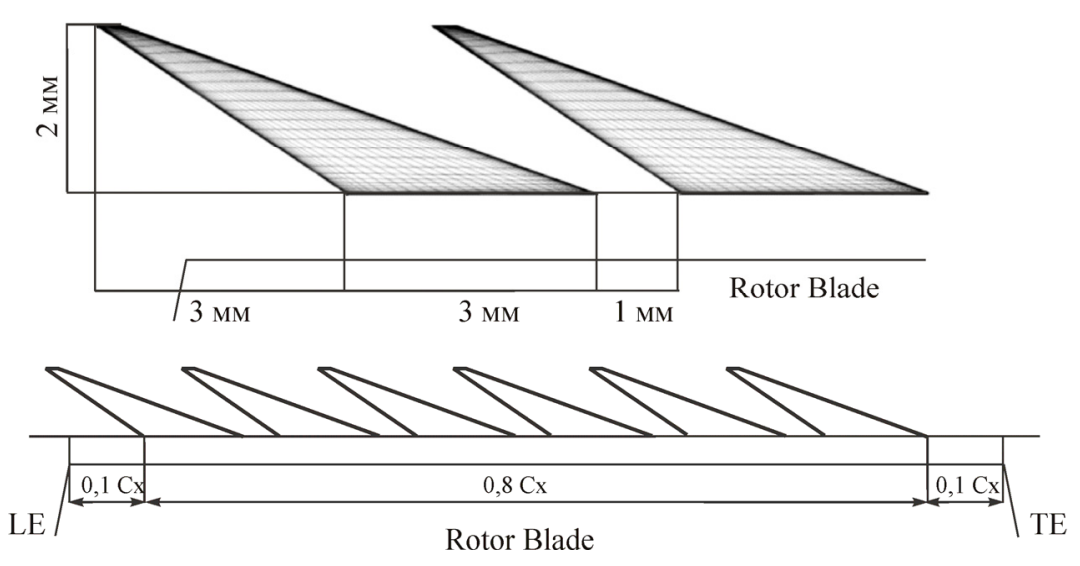

$a$
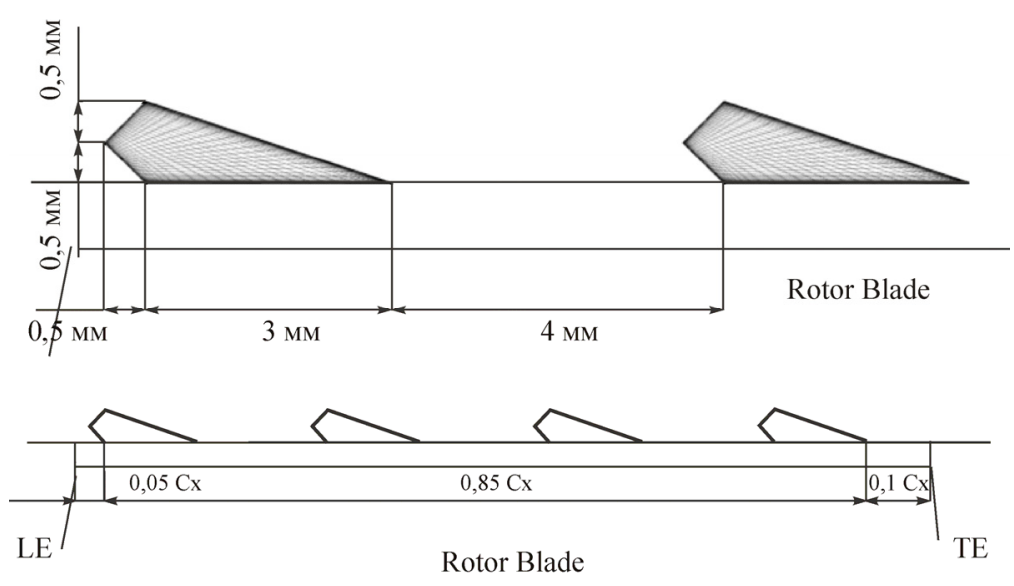

$\sigma$

Рис. 5. Конфигурации НРУ лабиринтного типа: $a$ - для расчетной модели; $\sigma$ - после учета технологических рекомендаций

\section{Результаты расчетов}

В результате серии расчетов были получены интегральные характеристики, показанные на рис. 6-8. При величине радиального зазора 0,4 мм влияние бороздок практически отсуствует. Увеличение радиального зазора до 0,8 мм повлекло за собой падение КПД примерно на 1,5\% как в случае варианта без статора, так и в случае полной ступени. Однако при остутсвии статора увеличение радиального зазора также привело к падению запасов газодинамической устойчивости, что не наблюдается в случае полной ступени. В обоих вариантах применение НРУ лабиринтного типа позволило вернуть часть КПД, а в случае ступени без статора также восстановить запасы устойчивости.

На рис. 6 продемонстрировано сравнение расчетных (см. рис. 6, $a$ ), и экспериментальных (см. рис. 6, б) данных по степени повышения полного давления $\pi^{*}$ и коэффициенту полезного действия $\eta_{\text {ад }}^{*}$ в зависимости от приведенного расхода для ступени Д-77М в компоновке без выходного статора CAII. Сравнение расчетных и экспериментальных данных показывает вполне удовлетворительное их соответствие как для варианта ступени с гладкой проточной частью при радиальных зазорах $d_{\mathrm{p}}=0,4$ мм и $d_{\mathrm{p}}=0,8$ мм, так и при наличии НРУ с числом бороздок $z=4$ при радиальном зазоре $d_{\mathrm{p}}=0,8$ мм. 

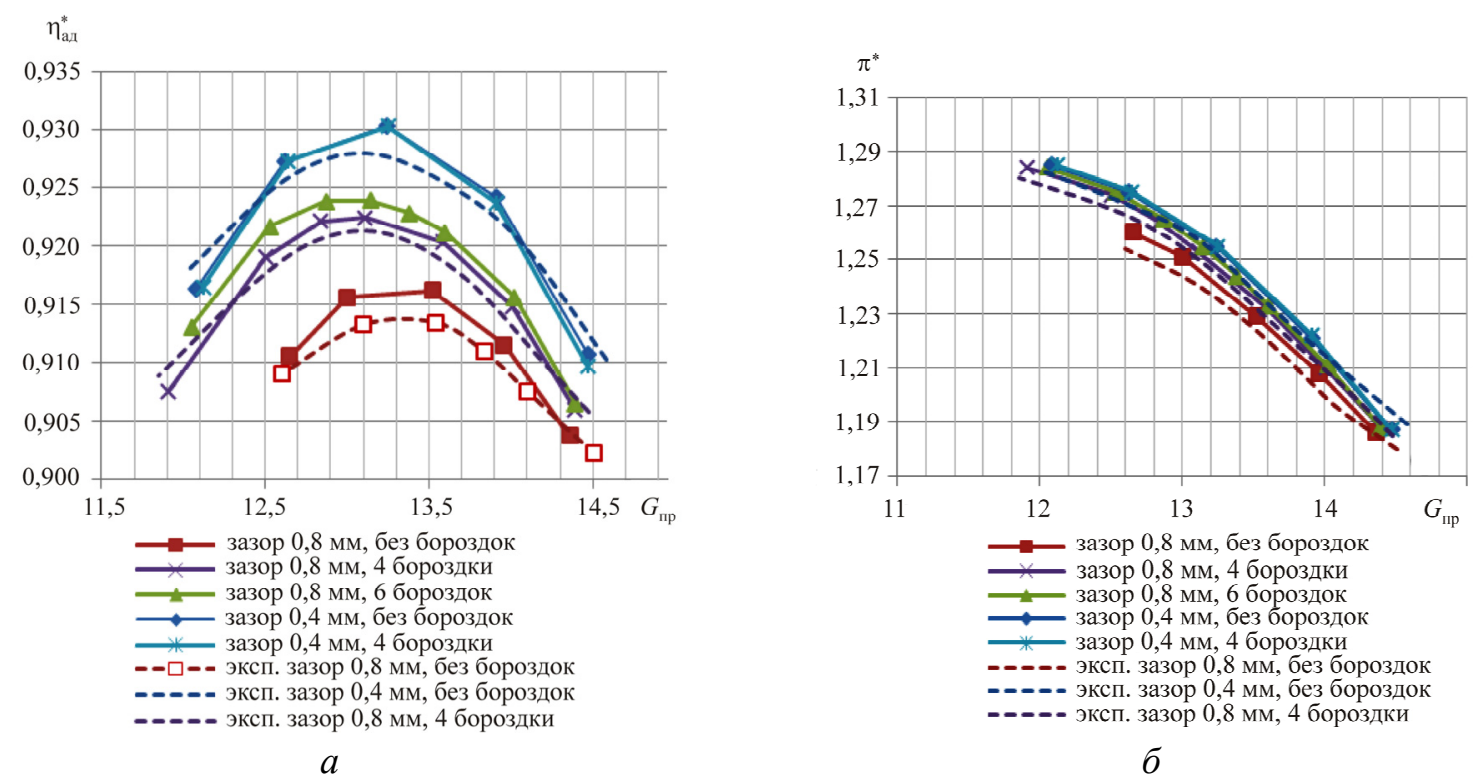

Рис. 6. Интегральные характеристики ступени без выходного статора (ВНА + РК), mixing plane в координатах: $a-\eta_{\text {ад }}^{*}\left(G_{\text {пр }}\right) ; \sigma-\pi^{*}\left(G_{\text {пр }}\right)$

На рис. 7 показаны интегральные характеристики ступени Д-77М в компоновке без выходного статора CAII, полученные с использованием метода NLH. Такая постановка была выбрана для подтверждения результатов, полученных с использованием приближения mixing plane. Расчеты методом NLH требуют больших затрат времени, поэтому данным методом была исследована только одна комбинация НРУ лабиринтного типа.

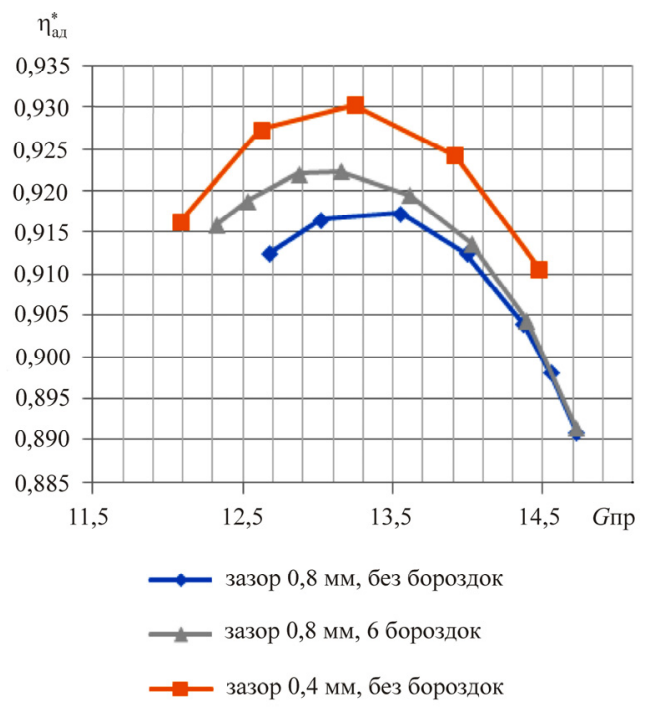

$a$

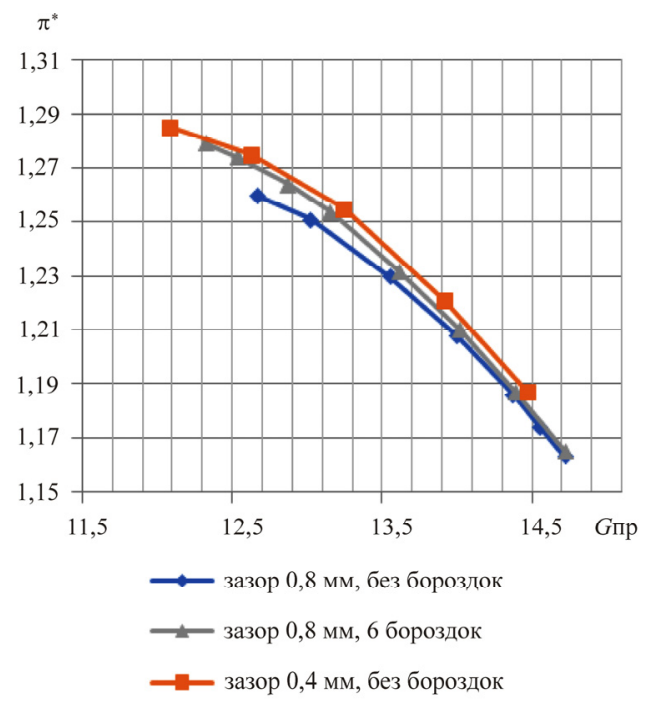

6

Рис. 7. Интегральные характеристики ступени без выходного статора (BHA + PK), NLH в координатах:

$$
a-\eta_{\text {ад }}^{*}\left(G_{\text {пр }}\right) ; \sigma-\pi^{*}\left(G_{\text {пр }}\right)
$$

На рис. 8 показаны интегральные характеристики ступени Д-77М в компоновке $\mathrm{BHA}+\mathrm{PK}+\mathrm{CA}_{\mathrm{II}}$, полученные в расчете с использованием приближения mixing plane. Точно так же, как и для ступени без статора, исследуемые НРУ лабиринтного типа не оказывают видимого эффекта на интегральные характеристики ступени в этой компоновке при номинальном радиальном зазоре. Увеличение зазора в 2 раза приводит к падению КПД на 1,5 \%. Применение НРУ позволяет вернуть 0,5 \% КПД, но это не приводит к увеличению запасов ГДУ. Экспери- 
ментальные характеристики для полной ступени не получены, так как исходный статор не удовлетворял требованиям. Он был перепроектирован и на данный момент изготавливается. Сравнение характеристик полной ступени будет следующим шагом данной работы.

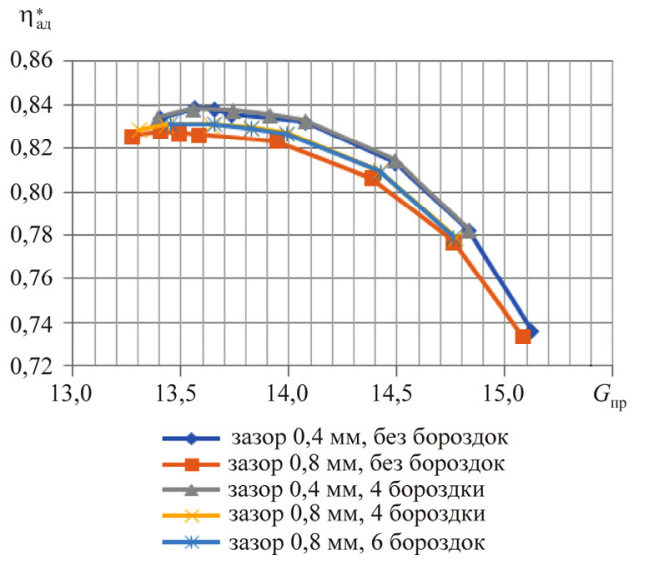

$a$

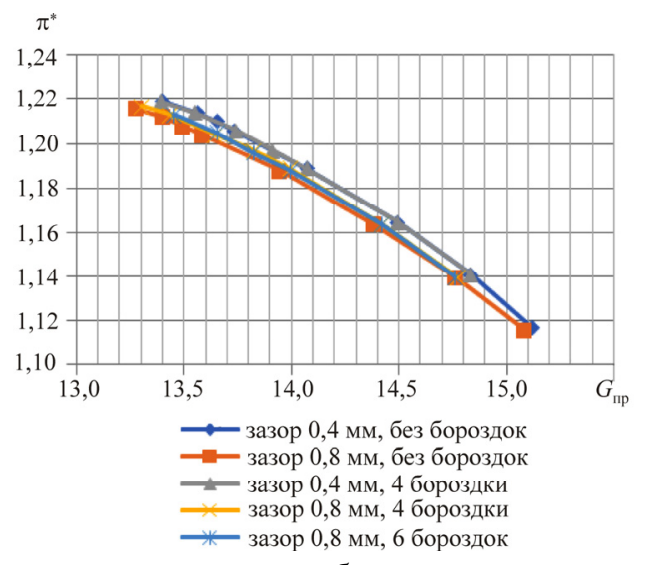

6

Рис. 8. Интегральные характеристики полной ступени (BHA + PK $+\mathrm{CA}_{\text {II }}$ ), mixing plane в координатах:

$$
a-\eta_{\text {ад }}^{*}\left(G_{\text {пр }}\right) ; \sigma-\pi^{*}\left(G_{\text {пр }}\right)
$$

На рис. 9 представлено распределение осредненной радиальной компоненты с целью выделить линии тока вблизи границы ГДУ. При величине радиального зазора 0,4 мм обратные токи занимают узкую область на периферии лопатки ближе к передней кромке. При увеличении радиального зазора до 0,8 мм область обратных токов заметно разрастается как в ширину, так и в глубину. Наступление срыва напрямую связано с размерами и положением зоны обратных токов от перетекания через радиальный зазор. Применение НРУ лабиринтного типа позволяет существенно уменьшить размеры этой зоны. Вместо единого центра теперь имеется несколько более мелких образований, которые располагаются под бороздками. Таким образом, контролируя рост зоны обратных токов при увеличении радиального зазора, можно добиться повышения запасов ГдУ.

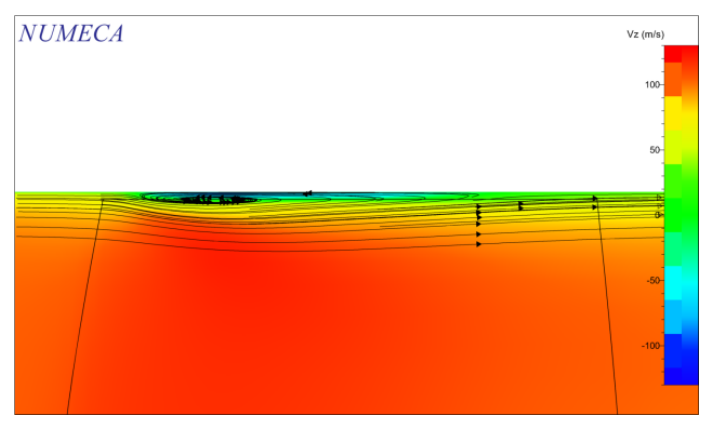

$a$

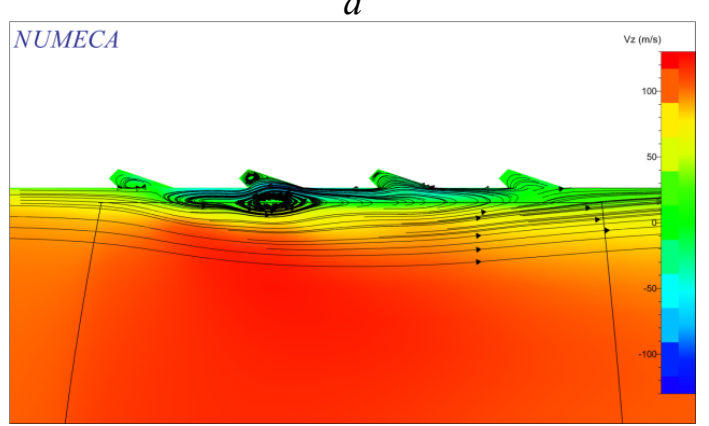

8

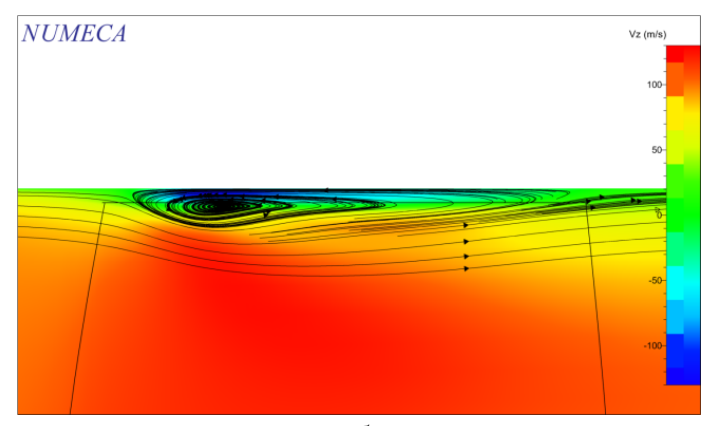

6

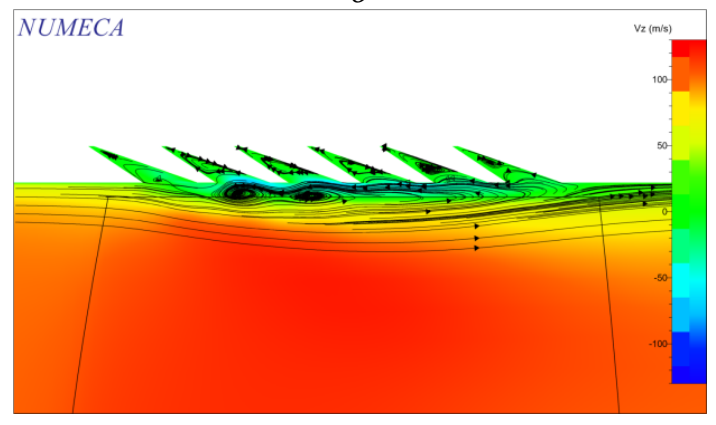

2

Рис. 9. Распределение радиальной компоненты скорости, осредненной в окружном направлении (меридиональное сечение, граница ГДУ, mixing plane): $a$ - ВНА + РК, радиальный зазор 0,4 мм, без НРУ; б-ВНА + РК, радиальный зазор 0,8 мм, без НРУ; в-ВНА + РК, радиальный зазор 0,8 мм, НРУ 4 бороздки; г- ВНА + РК, радиальный зазор 0,8 мм, НРУ 6 бороздок 
В рабочей точке (рис. 10) область обратных токов меньше и смещена ближе к центру лопатки. Более высокий прирост эффективности от применения шести бороздок объясняется тем, что бороздки, расположенные кучнее, разбивают вихрь на несколько более мелких. Тем не менее применение четырех бороздок НРУ также имеет положительный эффект.

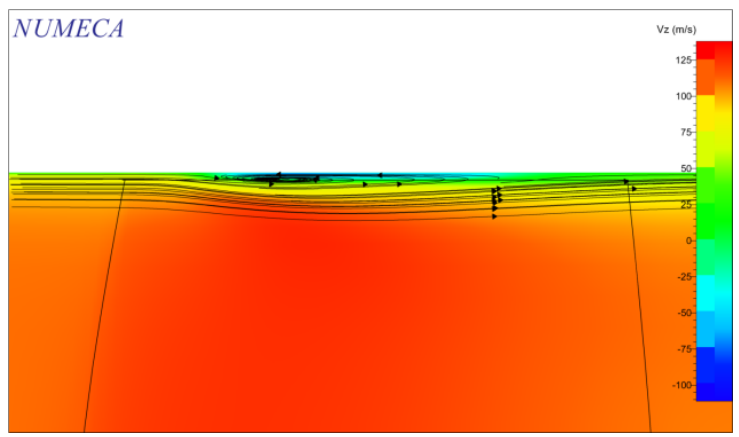

$a$

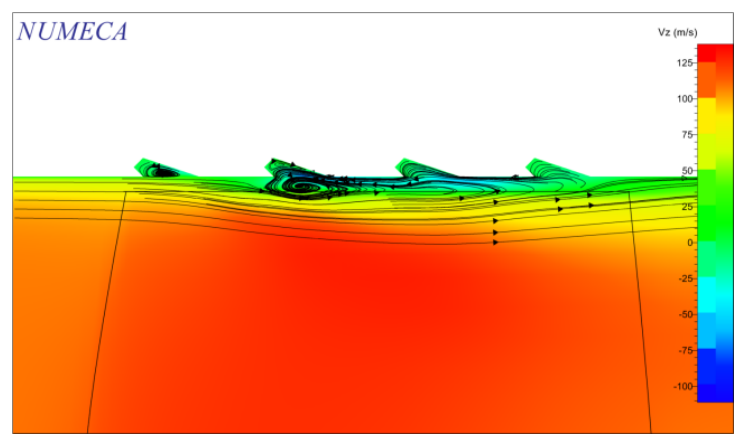

B
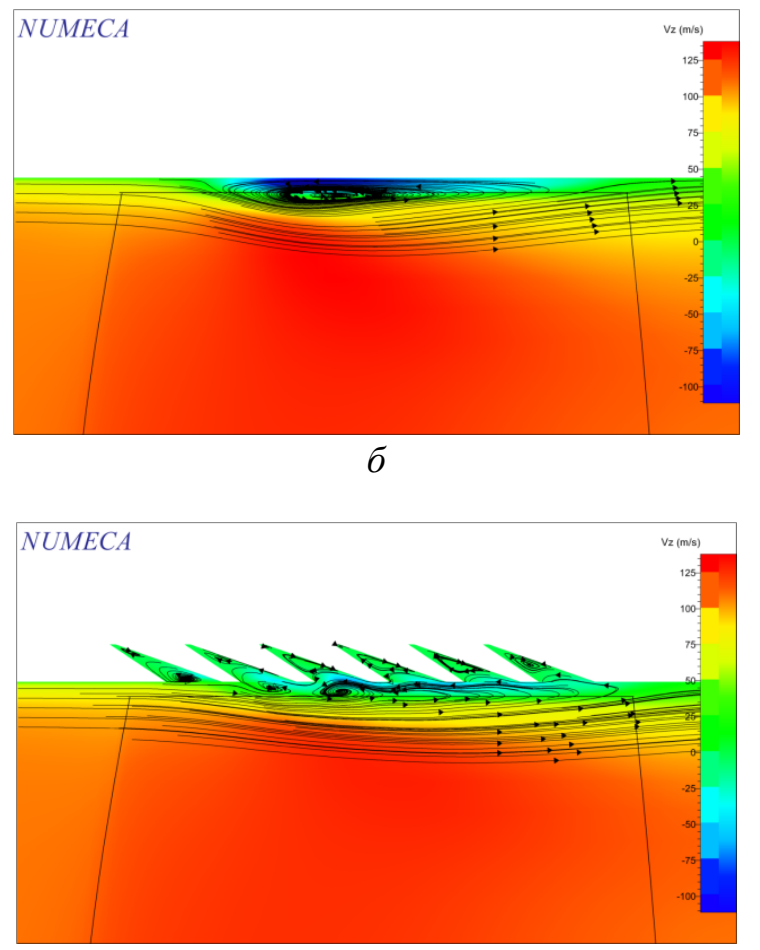

Рис. 10. Распределение радиальной компоненты скорости, осредненной в окружном направлении (меридиональное сечение, рабочая линия, mixing plane): $a$ - ВНА + РК, радиальный зазор 0,4 мм, без НРУ; б-ВНА + РК, радиальный зазор 0,8 мм, без НРУ; в-ВНА + РК, радиальный зазор 0,8 мм, НРУ 4 бороздки; г - ВНА + РК, радиальный зазор 0,8 мм, НРУ 6 бороздок

На рис. 11, $a, \sigma$ в распределении изолиний чисел Маха при зазоре 0,4 мм наблюдается застойная зона с малыми относительными числами Маха со стороны корытца лопатки, в задней ее части. При увеличении зазора до 0,8 мм размеры этой зоны также увеличиваются. Применение НРУ лабиринтного типа позволяет практически полностью убрать эту область, смягчая негативные последствия увеличения радиального зазора [23, 24], что показано на рис. $11,6,2$.

На рис. 12 показана структура вихря перетекания через радиальный зазор. На рис. 12, $a$ в варианте без НРУ с передней кромки сходит вихрь от перетекания. На рис. 12, $\sigma$ в варианте НРУ с четырьмя бороздками интенсивность этого вихря заметно ниже и его траектория располагается ближе к стороне разрежения лопатки, что хорошо согласуется с предыдущими исследованиями [9].

На рис. 13 показано, как из-за перетекания через радиальный зазор возникает и изменяется застойная зона у стороны давления лопатки на периферии. Положение этой зоны совпадает с траекторией вихря перетекания. Применение НРУ лабиринтного типа позволяет добиться значительного уменьшения этой зоны путем снижения интенсивности перетекания через радиальный зазор.

Увеличение интенсивности вихря перетекания приводит к производству энтропии (рис. 14) и генерации потерь. Применение НРУ позволяет заметно снизить производство энтропии и, как следствие, получить прирост КПД. 


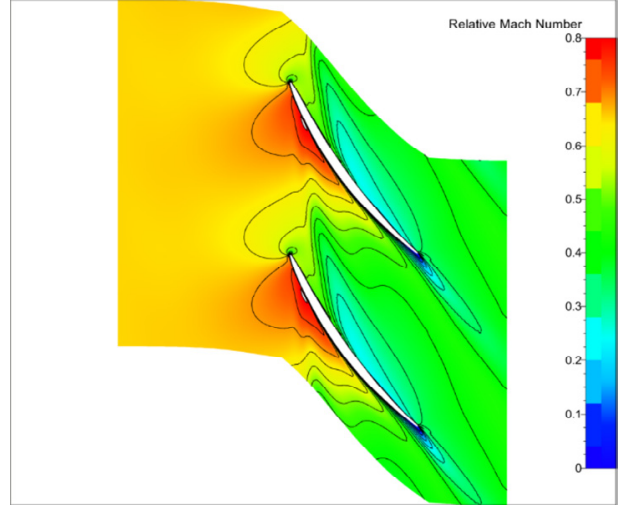

$a$

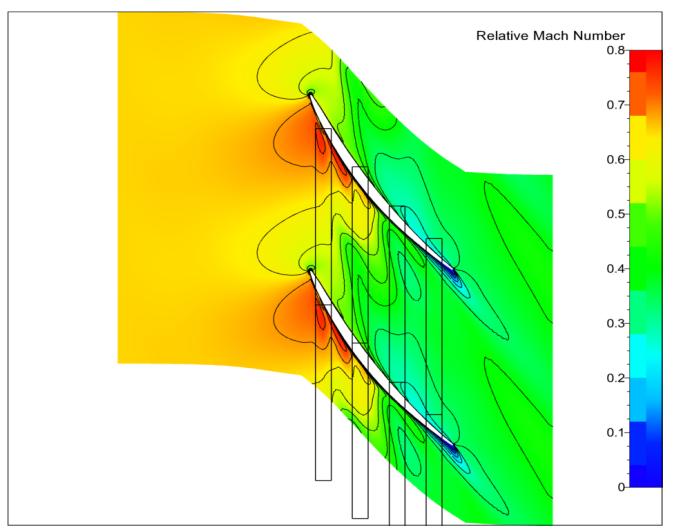

B
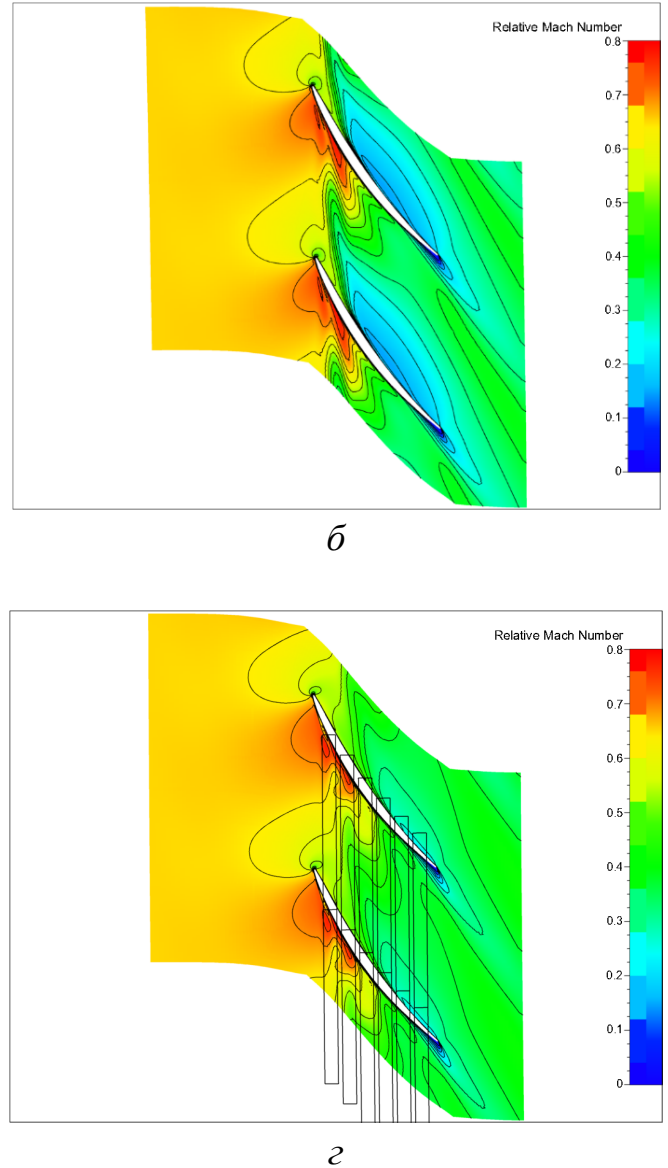

Рис. 11. Распределение чисел Маха в относительной системе координат (сечение межлопаточного канала на высоте 0,97h, граница ГДУ, mixing plane): $a$ - ВНА + РК, радиальный зазор 0,4 мм, без НРУ;

$\sigma$ - ВНА + РК, радиальный зазор 0,8 мм, без НРУ; в-ВНА + РК, радиальный зазор 0,8 мм, НРУ 4 бороздки; г - ВНА + РК, радиальный зазор 0,8 мм, НРУ 6 бороздок

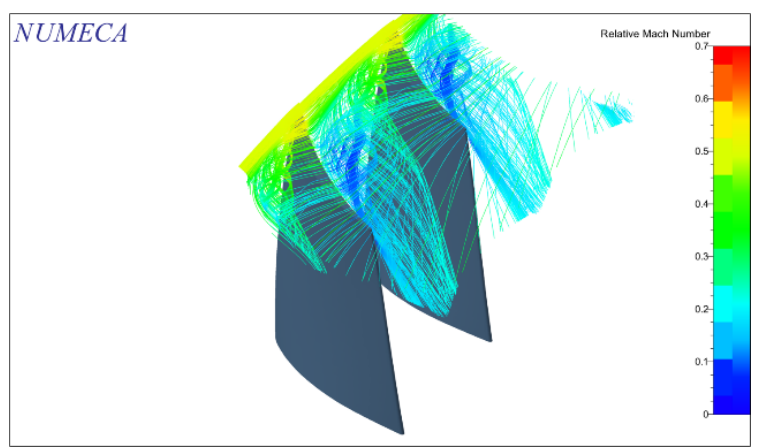

a

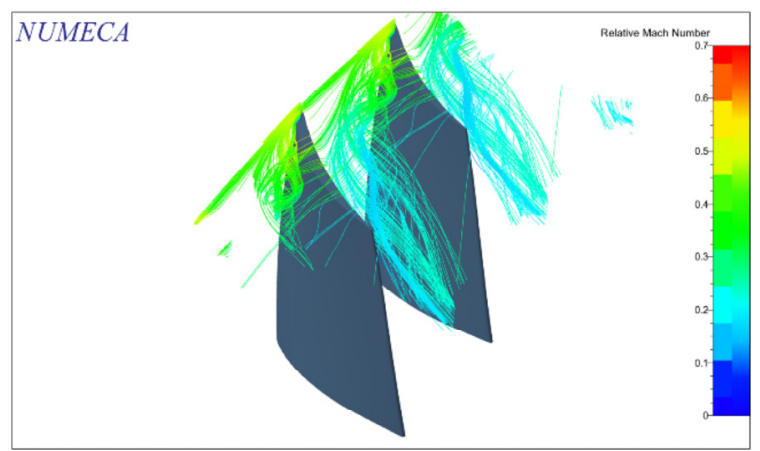

6

Рис. 12. Распределение чисел Маха в относительной системе координат (сечения в межлопаточном канале, граница ГДУ, mixing plane): $a$ - ВНА + РК, радиальный зазор 0,4 мм, без НРУ; $\sigma$ - ВНА + РК, радиальный зазор 0,8 мм, НРУ 4 бороздки 

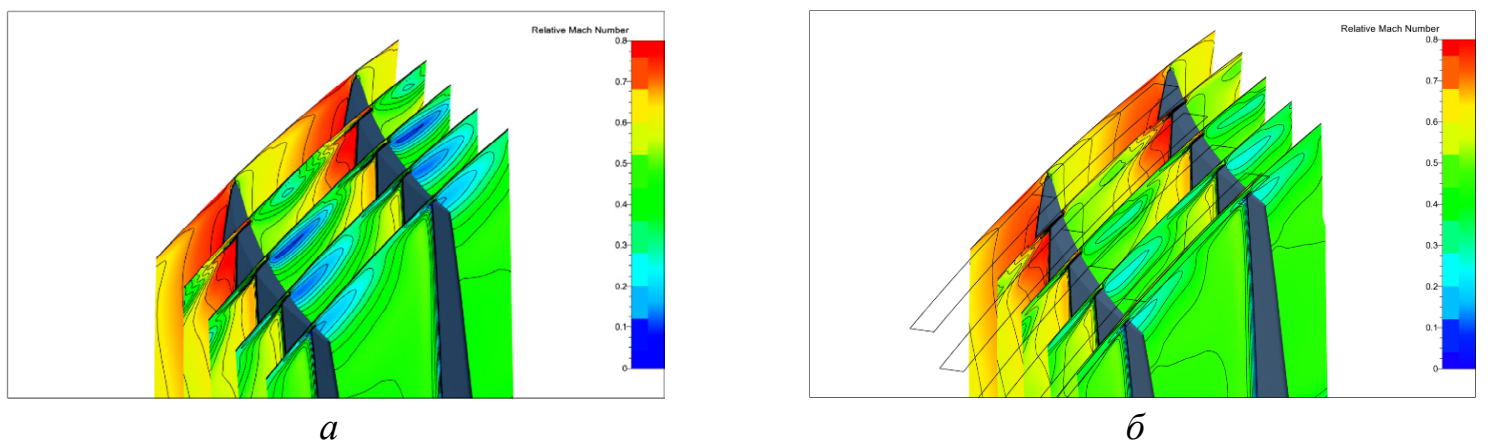

Рис. 13. Распределение чисел Маха в относительной системе координат (сечение межлопаточного канала на высоте 0,97h, граница ГДУ, NLH): $a$ - ВНА + РК, радиальный зазор 0,8 мм, без НРУ; $\sigma$ - ВНА + РК, радиальный зазор 0,8 мм, НРУ 6 бороздок
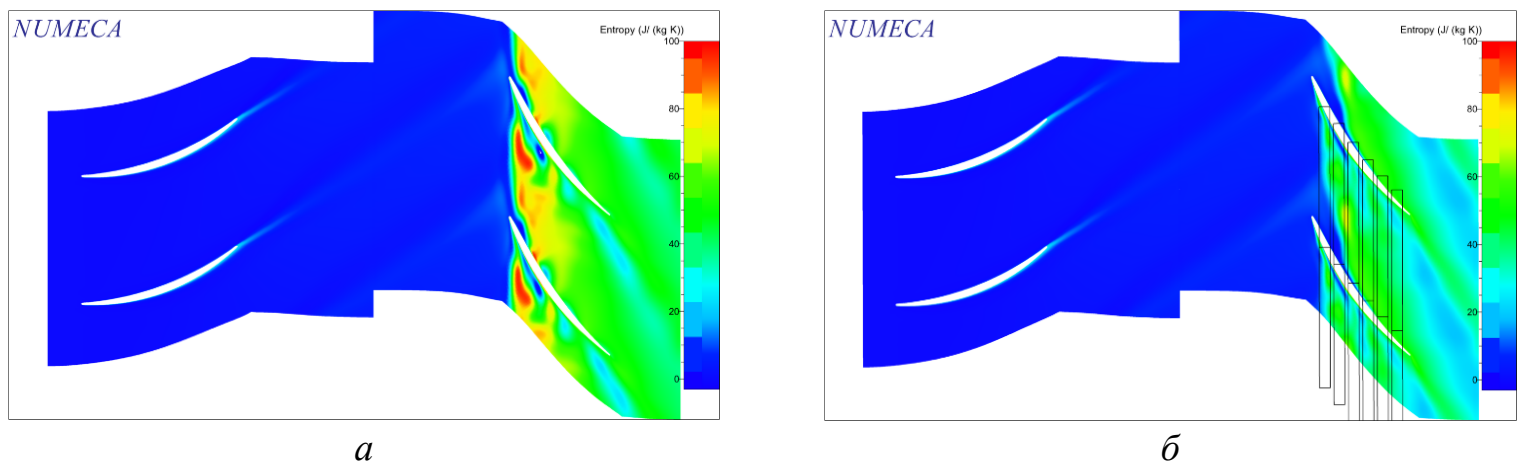

Рис. 14. Распределение энтропии (сечение межлопаточного канала на высоте $0,97 h$, граница ГДУ, NLH): $a$ - ВНА + РК, радиальный зазор 0,8 мм, без НРУ; $\sigma$ - ВНА + РК, радиальный зазор 0,8 мм, НРУ 6 бороздок

На рис. 15 показано распределение относительных чисел Маха на периферии для полной ступени. Застойная зона в задней части лопатки рабочего колеса значительно меньше, чем в случае, когда статор не включен в расчетную область. Тем не менее НРУ лабиринтного типа практически полностью убирает эту застойную зону. В области передней кромки заметно перестраивается структура вихря перетекания. Также стоит отметить, что НРУ практически не влияет на течение в последующих венцах.

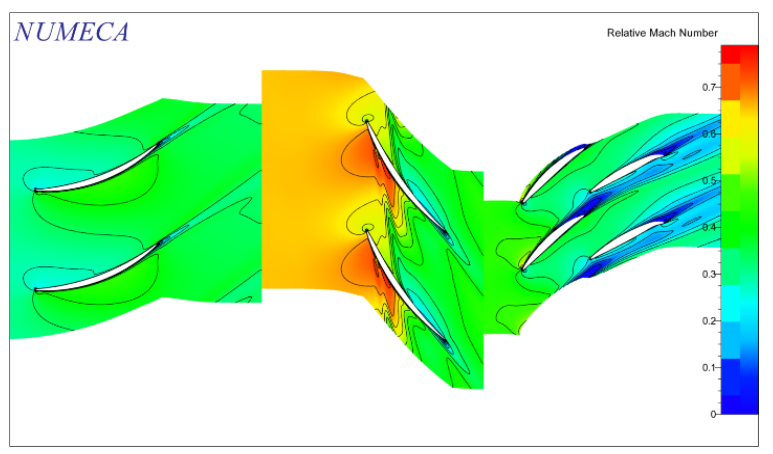

$a$

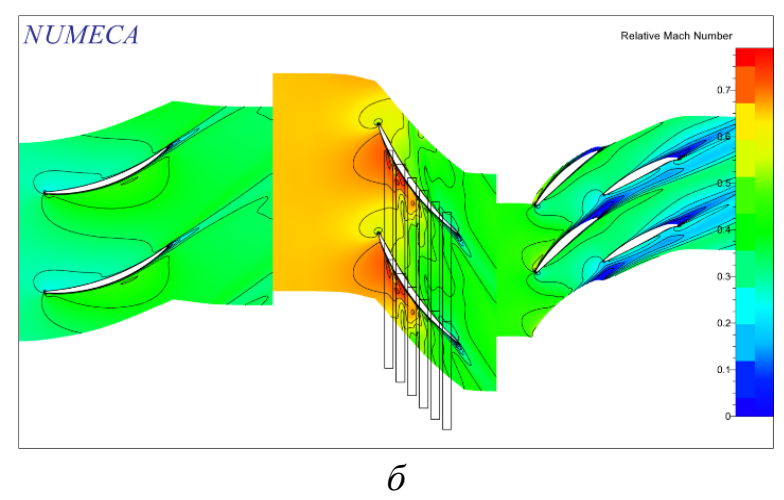

Рис. 15. Распределение чисел Маха в относительной системе координат (сечение межлопаточного канала на высоте 0,97h, граница ГДУ, полная ступень, mixing plane): $a$ - ВНА + PК + CA без НРУ; $\sigma$ - ВНА + РК + СА І, радиальный зазор 0,8 мм, НРУ 6 бороздок 
Особенностью замыкающей ступени

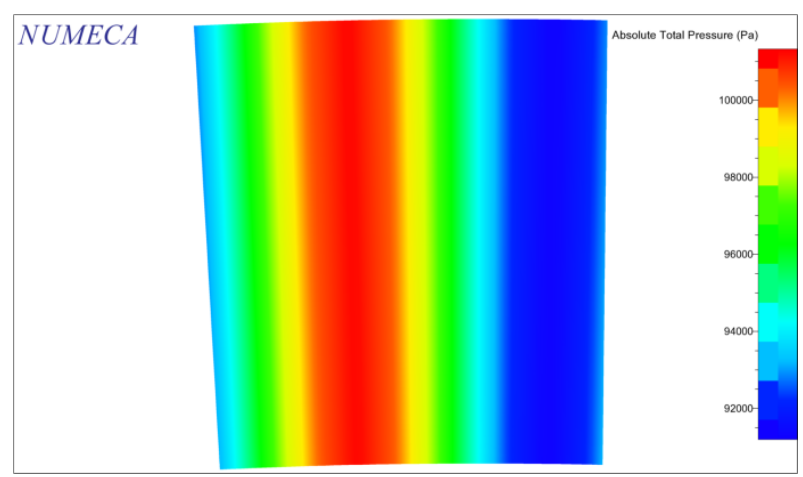

Рис. 16. Форма входного следа высоконагруженного КВД является мощное следовое возмущение, приходящее от предыдущих ступеней. Нестационарное взаимодействие этого возмущения и рабочего колеса существенно меняет картину течения, и эффективность НРУ в такой ситуации оказывается под вопросом [25]. В данной работе было численно смоделировано следовое возмущение путем подачи на вход провала полного давления на $10 \%$ (рис. 16) и показано, что и в такой постановке НРУ лабиринтного типа имеет также положительный эффект на характеристики замыкающей ступени компрессора.

На рис. 17 показано сравнение интегральных характеристик ступени Д-77М в компоновке без выходного статора $\mathrm{CA}_{\text {II }}$ при радиальном зазоре над PK 0,8 мм, полученных методом NLH при задании на входе равномерного потока и потока с провалом полного давления, моделирующего окружную неравномерность потока. При наличии входной неравномерности эффект НРУ сохранился: КПД вырос незначительно, а запасы ГДУ увеличились на 4,6 \%.
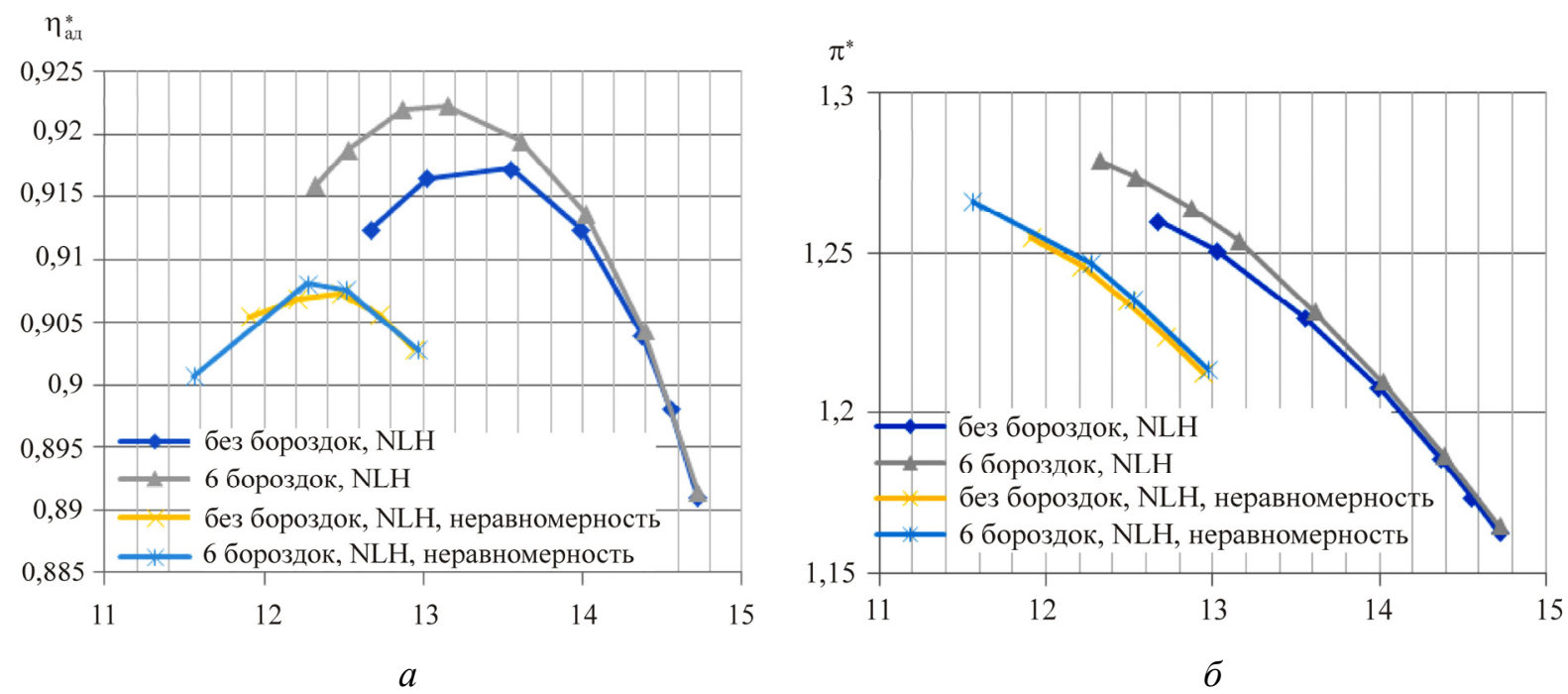

Рис. 17. Интегральные характеристики ступени без выходного статора (ВНА + РК) в координатах: $a-\eta_{\text {ад }}^{*}\left(G_{\text {пр }}\right) ; \sigma-\pi^{*}\left(G_{\text {пр }}\right)$, радиальный зазор 0,8 мм, NLH, неравномерность

\section{Выводы}

1. Проведены расчеты 3D-вязких турбулентых течений в замыкающей ступени Д-77М с гладкой проточной частью и несколькими вариантами НРУ лабиринтного типа в случаях, когда спрямляющий аппарат был отброшен и когда он входил в расчетную область. При величине радиального зазора 0,4 мм надроторные устройства лабиринтного типа не оказали влияния на характеристики ступени в обоих случаях. При увеличении зазора до 0,8 мм эффект НРУ стал заметен. Наибольший прирост дала конфигурация НРУ лабиринтного типа с шестью бороздками треугольной формы. Запасы ГДУ ступени без выходного спрямляющего аппарата с такой конфигурацией бороздок возросли на $7 \%$, а максимальный КПД на $0,8 \%$. В случае полной ступени конфигурация с шестью бороздками дала прирост КПД на $0,4 \%$ без изменения запасов ГДУ. 
2. Получены стационарные и нестационарные поля течения в межлопаточных каналах ступени Д-77М с гладкой проточной частью и с НРУ лабиринтного типа.

3. Показано, что применение НРУ лабиринтного типа позволяет снижать интенсивность вихря перетекания через радиальный зазор, тем самым улучшая обтекание лопатки в периферийной области и увеличивая запасы ГДУ.

\section{Библиографический список}

1. Dr. H. Scheugenpflug, Dr. Aspi Wadia. Technologies for the Next Engine Generation GE Aviation // Proc. of XXI Inter. Sym. on Air Breath. Engines, Seoul, South Korea. - 2013. - Invited lecture ISABE2013-1007. P. $1-23$.

2. Prahst P.S., Kulkarni S., Sohn Ki.H. Experimental results of the first two stages of an advanced transonic core compressor under isolated and multi-stage conditions // Proc. of ASME Turbo Expo 2015. - 2015. Paper No. GT2015-42727. - 11 p.

3. Lurie D.P., Breeze-Stringfellow A. Evaluation of experimental data from a highly loaded transonic compressor stage to determine loss sources // Proc. of ASME Turbo Expo 2015. - 2015. - Paper No. GT201542526. - 13 p.

4. Mileshin V.I. Numerical and experimental investigation of bypass fan stage models and high loaded compressor stages for development of new fan and high pressure compressor for advanced engines // Proc. of 10 Europ. Turbomachinery Conf. 2013, Lappeenranta, Finland, Invited lecture. - P. 1-58.

5. Mileshin V.I. Key-note speech "Challenges in fan and high pressure compressor development" // Proc. of XXI Inter. Symp. on Air Breath. Engines, Busan, South Korea. - Paper No. ISABE2013-1003. - P. 1-49.

6. Effect of tip clearance on flow structure and integral performances of six-stage HPC / V.I. Mileshin, I.K. Orekhov, V.A. Fateyev, S.K. Shchipin // Proc. of XVIII Inter. Symp. on Air Breath. Engines, Beijing, China. - 2007. - Paper No. ISABE-2007-1179. - 82 p.

7. Numerical and experimental analysis of radial clearance influence on rotor and stator clocking effect by example of model high loaded two stage compressor / V.I. Mileshin, N.M. Savin, P.G. Kozhemyako, Ya.M. Druzhinin // Proc. of ASME Turbo Expo 2014, Dusseldorf, Germany. - 2014. - Paper No. GT2014-26345. - Vol. 2a. - P. V02AT37A037.

8. Stall margin improvement in three-stage low pressure compressor by use of slot type casing treatments / F.Sh. Gelmedov, V.I. Mileshin, P.G. Kozhemyako, I.K. Orekhov // Proc. of ASME Turbo Expo 2014, Dusseldorf, Germany. - 2014. - Paper No. GT2014-26298. - P. V02AT37A036. - 11 p. DOI: 10.1115/GT2014-26298

9. Mileshin V.I., Brailko I., Startsev A. Application of casing circumferential grooves to counteract the influence of tip clearance // Proc. of ASME Turbo Expo. - 2008. - Paper No. GT2008-51147. - P. 617-627. - 11 p. DOI: $10.1115 /$ GT2008-51147

10. Shraman Narayan Goswami, Prof. M. Govardhan. Effect of sweep on performance of an axial compressor with casing grooves // Proc. of ASME Turbo Expo 2016, Seoul, South Korea. - 2016. - Paper No. GT2016-56045. - 10 p.

11. A study of performance and flow mechanism of a slot-groove hybrid casing treatment in a low-speed compressor / Juan Du, Fan Li, Jichao Li, Ning Ma, Feng Lin, Jingyi Chen // Proc. of ASME Turbo Expo 2015, Montreal, Canada. - 2015. - Paper No. GT2015-43920. - P. V02CT44A029. - 10 p. DOI: 10.1115/GT201543920

12. Wilke I., Kau H.-P. A numerical investigation of the flow mechanisms in a hpc front stage with axial slots // Proc. of ASME Turbo Expo 2003, Atlanta, USA. - 2003. - Paper No. GT2003-38481. - P. 465-477. 13 p. DOI: $10.1115 /$ GT2003-38481

13. Parametric study of tip clearance - casing treatment on performance and stability of a transonic axial compressor / B.H. Beheshti, J.A. Teixeira, P.C. Ivey, K. Ghorbainian, B. Farhanieh // Proc. of ASME Turbo Expo 2004, Vienna, Austria. - 2004. - Paper No. GT2004-53390. - P. 395-404. - 10 p. DOI: $10.1115 /$ GT2004-53390

14. Muller M.W., Schiffer H.-P., Hah C. Effect of circumferential grooves on the aerodynamic performance of an axial single-stage transonic compressor // Proc. of ASME Turbo Expo 2007, Montreal, Canada. 2007. - Paper No. GT2007-27365. - P. 115-124. - 10 p. DOI: 10.1115/GT2007-27365

15. Rabe D.C., Hah C. Application of casing circumferential grooves for improved stall margin in a transonic compressor // Proc. of ASME Turbo Expo 2002, Amsterdam, The Netherlands. - 2002. - Paper No. GT2002-30641. - P. 1141-1153. - 13 p. DOI: 10.1115/GT2002-30641 
16. Shabbir A., Adamzyk J.J. Flow mechanism for stall margin improvement due to circumferential casing grooves on axial compressor // Proc. of ASME Turbo Expo 2004, Vienna, Austria. - 2004. - Paper No. GT2004-53903. - P. 557-569. - 13 p. DOI: 10.1115/GT2004-53903

17. Georgios Goinis, Christian Voß, Marcel Aulich. Circumferential grooves for a modern transonic compressor: aerodynamic effects, benefits and limitations // Proc. of 10 Europ. Turbomachinery Conf. 2013, Lappeenranta, Finland. - Paper No. ETC10-60. - P. 1-14.

18. Rolfes M., Lange M., Vogeler K. Experimental investigation of circumferential groove casing treatments for large tip clearances in a low speed axial research compressor // Proc. of ASME Turbo Expo 2015, Montreal, Canada. - 2015. - Paper No. GT2015-42646. - P. V02AT37A021. - 10 p. DOI: 10.1115/GT201542646

19. Experimental and numerical investigation of effect of centeroffset degree on compressor stability with circumferential grooved casing treatment / Hao Guang Zhang, Feng Tan, Yan Hui Wu, Wu Li Chu, Wei Wang, Kang An // Proc. of ASME Turbo Expo 2016, Seoul, South Korea. - 2016. - Paper No. GT2016-56757. P. V02AT37A021. - 9 p. DOI: 10.1115/GT2016-56757

20. Hirsch Ch. Numerical computation of internal and external flows // John Wiley \& Sons. - 1990. Vol. 2. - P. 691.

21. Hirsch Ch. Non-deterministic methodologies for uncertainty quantification in turbomachinery CFD, Numeca international, Brussels. Pannel session // Proc. of ASME Turbo Expo 2012, Copenhagen, Denmark. 2012. - P. 1-18.

22. Recommendations for achieving accurate numerical simulation of tip clearance flows in transonic compressor rotors / Van Zante [et al.] // ASME J. Turbomach. - 1999. - No. 122(4). - P. 733-742. 10 p. DOI: $10.1115 / 1.1314609$

23. Casing treatment for desensitization of compressor performance and stability to tip clearance / M. Cevik [et al.] // ASME J. Turbomach. - 2016. - Vol. 138(12). - Paper No. TURBO-15-1242. - 16 p. DOI: $10.1115 / 1.4033420$

24. Desensitization of axial compressor performance and stability to tip clearance size / E. Erler [et al.] // ASME J. Turbomach. - 2015. - Vol. 138(3). - Paper No. TURBO-15-1177. - 12 p. DOI: 10.1115/1.4031865

25. He L., Ning W. Efficient approach for analysis of unsteady viscous flows in turbomachines // AIAA J. - 1998. - Vol. 36. - No. 11. - P. 2005-2012.

\section{Reference}

1. Dr. H. Scheugenpflug and Dr. Aspi Wadia. Technologies for the Next Engine Generation GE Aviation // Proceedings of XXI International Symposium on Air Breathing Engines, Seoul, South Korea, Invited lecture ISABE2013-1007, 2013, pp. 1-23.

2. P.S. Prahst, S. Kulkarni, Ki H. Sohn, Experimental results of the first two stages of an advanced transonic core compressor under isolated and multi-stage conditions // Proceedings of ASME Turbo Expo 2015, Paper No. GT2015-42727, 2015, 11 рю

3. D.P. Lurie, A. Breeze-Stringfellow. Evaluation of experimental data from a highly loaded transonic compressor stage to determine loss sources // Proceedings of ASME Turbo Expo 2015, Paper No. GT2015-42526, 2015, 13 p.

4. V.I. Mileshin. Numerical and experimental investigation of bypass fan stage models and high loaded compressor stages for development of new fan and high pressure compressor for advanced engines // Proceedings of 10 European Turbomachinery Conference, Lappeenranta, Finland, Invited lecture, pp. 1-58.

5. V.I. Mileshin. Key-note speech "Challenges in fan and high pressure compressor development" // Proceedings of XXI International Symposium on Air Breathing Engines, Busan, South Korea, Paper No. ISABE2013-1003, pp. 1-49.

6. V.I. Mileshin, I.K. Orekhov, V.A. Fateyev, S.K. Shchipin. Effect of tip clearance on flow structure and integral performances of six-stage HPC // Proceedings of XVIII International Symposium on Air Breathing Engines, Beijing, China, Paper No. ISABE-2007-1179, 2007, 82 p.

7. V.I. Mileshin, N.M. Savin, P.G. Kozhemyako, Ya.M. Druzhinin. Numerical and experimental analysis of radial clearance influence on rotor and stator clocking effect by example of model high loaded two stage compressor // Proceedings of ASME Turbo Expo 2014, Dusseldorf, Germany, Paper No. GT2014-26345, 2014, vol. 2a, pp. V02AT37A037. 
8. F.Sh. Gelmedov, V.I. Mileshin, P.G. Kozhemyako, I.K. Orekhov. Stall margin improvement in threestage low pressure compressor by use of slot type casing treatments // Proceedings of ASME Turbo Expo 2014, Dusseldorf, Germany Paper No. GT2014-26298, pp. V02AT37A036, 11 p. DOI: 10.1115/GT2014-26298

9. Victor Mileshin, Igor Brailko, Andrew Startsev. Application of casing circumferential grooves to counteract the influence of tip clearance // Proceedings of ASME Turbo Expo 2008, No. GT2008-51147, pp. 617-627, 11 p. DOI:10.1115/GT2008-51147

10. Shraman Narayan Goswami, Prof. M. Govardhan. Effect of sweep on performance of an axial compressor with casing grooves // Proceedings of ASME Turbo Expo 2016, Seoul, South Korea, 2016, Paper No. GT2016-56045, 10 p.

11. Juan Du, Fan Li, Jichao Li, Ning Ma, Feng Lin, Jingyi Chen, A study of performance and flow mechanism of a slot-groove hybrid casing treatment in a low-speed compressor // Proceedings of ASME Turbo Expo 2015, Montreal, Canada, Paper No. GT2015-43920, pp. V02CT44A029, 10 p. DOI: $10.1115 / \mathrm{GT} 2015-43920$

12. Wilke, I., and Kau, H.-P. A Numerical Investigation of the Flow Mechanisms in a HPC Front Stage with Axial Slots // Proceedings of ASME Turbo Expo 2003, Atlanta, USA, Paper No. GT2003-38481, pp. 465-477, 13 p. DOI: 10.1115/GT2003-38481

13. Beheshti, B.H., Teixeira, J.A., Ivey, P.C., Ghorbainian, K., Farhanieh, B. Parametric Study of Tip Clearance - Casing Treatment on Performance and Stability of a Transonic Axial Compressor // Proceedings of ASME Turbo Expo 2004, Vienna, Austria, Paper No. GT2004-53390, pp. 395-404, 10 p. DOI: $10.1115 /$ GT2004-53390

14. Muller, M.W., Schiffer, H.-P. and Hah C. Effect of Circumferential Grooves on the Aerodynamic Performance of an Axial Single-Stage Transonic Compressor // Proceedings of ASME Turbo Expo 2007, Montreal, Canada, Paper No. GT2007-27365, pp. 115-124, 10 p. DOI: 10.1115/GT2007-27365

15. Rabe, D.C., and Hah, C. Application of Casing Circumferential Grooves for Improved Stall Margin in a Transonic Compressor // Proceedings of ASME Turbo Expo 2002, Amsterdam, The Netherlands, Paper No. GT2002-30641, pp. 1141-1153, 13 p. DOI: 10.1115/GT2002-30641

16. Shabbir A., and Adamzyk J.J. Flow Mechanism for Stall Margin Improvement due to Circumferential Casing Grooves on Axial Compressor // Proceedings of ASME Turbo Expo 2004, Vienna, Austria, Paper No. GT2004-53903, P. 557-569, 13 p. DOI: 10.1115/GT2004-53903

17. Georgios Goinis, Christian Voß and Marcel Aulich. Circumferential grooves for a modern transonic compressor: aerodynamic effects, benefits and limitations // Proceedings of 10 European Turbomachinery Conference, Lappeenranta, Finland, Paper No. ETC10-60, pp. 1-14.

18. M. Rolfes, M. Lange and K. Vogeler. Experimental investigation of circumferential groove casing treatments for large tip clearances in a low speed axial research compressor // Proceedings of ASME Turbo Expo 2015, Montreal, Canada, Paper No. GT2015, pp. V02AT37A021, 10 p. DOI: 10.1115/GT2015-42646

19. Hao Guang Zhang, Feng Tan, Yan Hui Wu, Wu Li Chu, Wei Wang, Kang An. Experimental and numerical investigation of effect of centeroffset degree on compressor stability with circumferential grooved casing treatment // Proceedings of ASME Turbo Expo 2016, Seoul, South Korea, Paper No. GT2016-56757, pp. V02AT37A021, 9 p. DOI: 10.1115/GT2016-56757

20. Hirsch Ch., 1990, Numerical Computation of Internal and External Flows. Volume 2 // John Wiley \& Sons, 1990, Vol. 2, 691 p.

21. Hirsch Ch. Non-Deterministic Methodologies for Uncertainty quantification in turbomachinery CFD, Numeca international, Brussels. Pannel session // Proceedings of ASME Turbo Expo 2012, Copenhagen, Denmark, pp. 1-18.

22. Van Zante et al. Recommendations for Achieving Accurate Numerical Simulation of Tip Clearance Flows in Transonic Compressor Rotors. ASME J. Turbomach, 1999, no. 122(4), pp. 733-742, 10 p. DOI: $10.1115 / 1.1314609$

23. Cevik, M. et al. Casing Treatment for Desensitization of Compressor Performance and Stability to Tip Clearance // ASME J. Turbomach., 2016, Vol. 138(12), 16 p., Paper No: TURBO-15-1242. DOI: $10.1115 / 1.4033420$

24. Erler, E. et al. Desensitization of Axial Compressor Performance and Stability to Tip Clearance Size // ASME J. Turbomach., 2015, Vol. 138(3), 12 p, Paper No: TURBO-15-1177. DOI: 10.1115/1.4031865.

25. He, L. and Ning, W. Efficient Approach for Analysis of Unsteady Viscous Flows in Turbomachines // AIAA Journal, 1998, Vol. 36, No. 11, pp. 2005-2012. 


\section{Об авторах}

Милешин Виктор Иванович (Москва, Россия) - кандидат физико-математических наук, начальник отделения ФГУП ЦИАМ им. П.И. Баранова (111116, г. Москва, ул. Авиамоторная, д. 2, е-таil: mileshin@ciam.ru).

Петровичев Александр Михайлович (Москва, Россия) - кандидат технических наук, начальник отдела ФГУП ЦИАМ им. П.И. Баранова (111116, г. Москва, ул. Авиамоторная, д. 2).

Баева Светлана Ивановна (Москва, Россия) - ведущий инженер ФГУП ЦИАМ им. П.И. Баранова (111116, г. Москва, ул. Авиамоторная, д. 2).

Жданов Владислав Вячеславович (Москва, Россия) - инженер, ФГУП ЦИАМ им. П.И. Баранова (111116, г. Москва, ул. Авиамоторная, д. 2, e-mail: vldzhdanov@yandex.ru).

\section{About the authors}

Viktor I. Mileshin (Moscow, Russian Federation) - CSc in Physical and Mathematical Sciences, Head of Division, Central Institute of Aviation Motors named after P.I. Baranov (CIAM) (2, Aviamotornaya st., Moscow, 111116, Russian Federation, e-mail: mileshin@ciam.ru).

Aleksandr M. Petrovichev (Moscow, Russian Federation) - CSc in Technical Sciences, Head of Department, Central Institute of Aviation Motors named after P.I. Baranov (CIAM) (2, Aviamotornaya st., Moscow, 111116, Russian Federation).

Svetlana I. Bayeva (Moscow, Russian Federation) - Leading Engineer, Central Institute of Aviation Motors named after P.I. Baranov (CIAM) (2, Aviamotornaya st., Moscow, 111116, Russian Federation).

Vladislav V. Zhdanov (Moscow, Russian Federation) - Engineer, Central Institute of Aviation Motors named after P.I. Baranov (CIAM) (2, Aviamotornaya st., Moscow, 111116, Russian Federation, e-mail: vldzhdanov@yandex.ru).

Получено 23.04.2019 